\title{
A NOTE ON QUASICENTRAL APPROXIMATE UNITS IN $B(H)$
}

\author{
JOHN L. ORR
}

(Communicated by John B. Conway)

\begin{abstract}
If a Hilbert Space, $H$, is infinite dimensional, $B(H)$ has no count-
\end{abstract} able quasicentral approximate unit for the ideal of finite rank operators.

Quasicentral approximate units were introduced in [2] by W. Arveson and independently by C. Akemann and G. K. Pedersen in [1]. Arveson has shown that for any $C^{*}$-algebra, $A$, quasicentral approximate units exist for all ideals of $A$. Further, if $A$ is a separable $C^{*}$-algebra, the quasicentral approximate unit may be taken to be an increasing sequence. While countable quasicentral approximate units can sometimes be found in the inseparable case, Arveson observes in [3] that it seems unlikely there is such an approximate unit for the ideal of finite rank operators in $B(H)$. Here we exhibit a proof of this fact.

Definition. Given a $C^{*}$-algebra, $A$, and an ideal, $K$, of $A$, an increasing net $\left\{u_{\lambda}\right\}$ of positive elements of $A$ is called an approximate unit for $K$ if $\left\|u_{\lambda}\right\| \leq 1$ and $\lim _{\lambda}\left\|u_{\lambda} k-k\right\|=0$ for all $k$ in $K$. An approximate unit is said, further, to be quasicentral if, for any $a$ in $A, \lim _{\lambda}\left\|u_{\lambda} a-a u_{\lambda}\right\|=0$.

Remarks. Recall that if $\left\{u_{\lambda}\right\}$ is an approximate unit for the ideal of finite rank operators in $B(H)$ then $\left\{u_{\lambda}\right\}$ must converge to the identity in the strong operator topology since $u_{\lambda} R-R$ converges to zero for each rank one operator $R$. Thus the following proposition suffices to show that $B(H)$ has no countable quasicentral approximate unit for the ideal of finite rank operators.

Throughout, we shall denote by $a \otimes b$ the rank one operator which maps $x$ to $\langle x, a\rangle b$ and assume that $H$ is an infinite dimensional Hilbert space.

Proposition. Let $\left(F_{n}\right)$ be an increasing sequence of positive finite rank operators in $B(H)$ and suppose $\left(F_{n}\right)$ converges to the identity, $I$, in the strong operator topology. Then there is a partial isometry, $U$, such that $F_{n} U-U F_{n}$ does not converge to zero in norm.

Proof. Since $0 \leq F_{1} \leq F_{2} \leq \cdots$, it follows that their ranges form an increasing sequence of subspaces. We can find an orthonormal sequence $\phi_{k}$

Received by the editors February 5, 1988.

1980 Mathematics Subject Classification (1985 Revision). Primary 46L05; Secondary 47C15.

The author wishes to acknowledge financial support from SERC (UK). 
and a sequence of integers, $k_{n}$, such that the range of each $F_{n}$ is the span $\left\{\phi_{1}, \ldots, \phi_{k_{n}}\right\}$. Clearly for each $i, \lim \left\langle F_{n} \phi_{i}, \phi_{i}\right\rangle=1$.

Now choose an integer $n_{1}$ such that $\left\langle F_{n_{1}} \phi_{1}, \phi_{1}\right\rangle \geq \frac{1}{2}$ and let $U_{1}=\phi_{1} \otimes$ $\phi_{k_{n_{1}+1}}$. Inductively obtain an increasing sequence, $n_{i}$, such that $\left\langle F_{n_{i}} \phi_{i}, \phi_{i}\right\rangle \geq \frac{1}{2}$ and $k_{n_{i}}>k_{n_{i-1}}$. Let $U_{i}=\phi_{i} \otimes \phi_{k_{n_{i}+1}}$ for each $i$. Since this forms a sequence of partial isometries with orthogonal intitial and final spaces, $U=\sum U_{i}$ is a partial isometry and $F_{n_{i}} U \phi_{i}=0$ for all $i$, since $U \phi_{i}$ is orthogonal to the range of the selfadjoint operator, $F_{n_{i}}$. Our result now follows from the inequalities below.

$$
\begin{aligned}
\left\|U F_{n_{i}}-F_{n_{i}} U\right\| & \geq\left|\left\langle\left(U F_{n_{i}}-F_{n_{i}} U\right) \phi_{i}, \phi_{k_{n_{i}+1}}\right\rangle\right| \\
& =\left|\left\langle U F_{n_{i}} \phi_{i}, \phi_{k_{n_{i}+1}}\right\rangle\right| \\
& =\left|\left\langle F_{n_{i}} \phi_{i}, \phi_{i}\right\rangle\right| \geq \frac{1}{2} \text { for each } i .
\end{aligned}
$$

\section{REFERENCES}

1. C. Akemann \& G. K. Pedersen, Ideal perturbations of elements of $C^{*}$-algebras, Math. Scand. 41 (1977), 117-139.

2. W. Arveson, Notes on extensions of $C^{*}$-algebras, Duke Math. J. 44 (1977), 329-355.

3. __ Perturbation theory for groups and lattices, J. Funct. Anal. 53 (1983), 22-73.

Department of Mathematics, King's College London, Strand, London WC2R 2LS EngLAND 